\title{
EFFECT OF THE COVID-19 EPIDEMIC ON PHYSICAL ACTIVITY IN COMMUNITY-DWELLING OLDER ADULTS IN JAPAN: A CROSS-SECTIONAL ONLINE SURVEY
}

\author{
M. YAMADA ${ }^{1}$, Y. KIMURA², D. ISHIYAMA², Y. OTOBE², M. SUZUKI², S. KOYAMA², T. KIKUCHI², \\ H. KUSUMI ${ }^{2}, \mathrm{H} . \mathrm{ARAI}^{3}$
}

\begin{abstract}
1. Faculty of Human Sciences, University of Tsukuba, Tokyo, Japan; 2. Graduate School of Comprehensive Human Sciences, University of Tsukuba, Tokyo, Japan; 3. National Center for Geriatrics and Gerontology, Aichi, Japan. Corresponding author: Minoru Yamada, PT, PhD, Faculty of Human Sciences, University of Tsukuba, 3-29-1 Otsuka, Bunkyo-ku, Tokyo 1120012, Japan, Tel: +81-3-3942-6863, Fax: +81-3-3942-6895, E-mail address: m-yamada@ human.tsukuba.ac.jp
\end{abstract}

\begin{abstract}
Objectives: The objective of this study was to investigate changes in physical activity (PA) between January (before the COVID-19 epidemic) and April (during the COVID-19 epidemic) 2020 in communitydwelling older adults in Japan. Design: Cross-sectional online survey. Setting and Subjects: From April 23 to 27, 2020, an online survey was completed by 1,600 community-dwelling older adults in Japan. Methods: We assessed the frailty status using the Kihon checklist, and other demographics and asked questions regarding PA at two time points: January and April 2020. We defined the total PA time (minutes) per week based on activity frequency and time. Results: The study participants' mean age, proportion of women, and prevalence of frailty were $74.0 \pm 5.6$ years, $50 \%(\mathrm{n}=800)$, and $24.3 \%(\mathrm{n}=388)$, respectively. We found a significant decrease in total PA time in April 2020 (median [interquartile range (IQR)], 180 [0 to 420]) when compared to January 2020 (median [IQR], 245 [90 to 480]) $(\mathrm{P}<0.001)$. We also performed a subgroup analysis according to the frailty category; total PA time significantly decreased in April 2020 when compared to January 2020 for all frailty categories $(\mathrm{P}<0.001)$. Conclusion: In conclusion, due to the COVID-19 epidemic, the total PA time in April 2020 significantly decreased compared to that in January 2020 in older adults. This finding may lead to a higher incidence of disability in the near future in older people.
\end{abstract}

Key words: COVID-19, physical activity, older adults.

\section{Introduction}

The Coronavirus disease 2019 (COVID-19) is creating a serious impact that causes a high morbidity and mortality rate worldwide. The World Health Organization (WHO) declared COVID-19 a pandemic in March 2020, and the Japanese government declared a state of emergency on April 7, 2020. In particular, older adults may be at high risk for severe illness from COVID-19, and several academic societies have proposed recommendations on COVID-19 for older adults. Many older adults, especially frail older adults, are more vulnerable to adverse health outcomes, such as falls and disabilities $(1,2)$.

Physical activity or exercise, such as walking, plays an important role in preventing several adverse health outcomes for older adults $(3,4)$. However, the amount of physical activity (PA) for older adults may notably decrease due to the COVID19 epidemic. In March 2011, the north-eastern part of Japan was rocked by a huge earthquake and tsunami. This disaster not only led to a large number of deaths, but also to a higher incidence of disability after a few months in disaster areas (5). One of the possible reasons is a decline in PA after a disaster, similarly, the COVID-19 epidemic may cause lower PA and a high incidence of disability.

The objective of this study, therefore, was to investigate the change in PA between January (before the COVID-19 epidemic) and April 2020 (during the COVID-19 epidemic) in community-dwelling Japanese older adults. We hypothesized that the PA in April has drastically decreased compared to that in January in older adults, especially for frail older adults.

\section{Methods}

\section{Participants}

From April 23 to 27, 2020, an online survey was completed by 1,600 community-dwelling older adults in Japan. The inclusion criteria included adults who were 65 to 84 years old without COVID-19 and were residents of Tokyo, Kanagawa, Chiba, Saitama, Aichi, Osaka, Hyogo, and Fukuoka prefectures, which are urban areas. This study was conducted in accordance with the guidelines proposed by the Declaration of Helsinki, and the study protocol was reviewed and approved by the Ethics Committee of the Faculty of Human Sciences, University of Tsukuba.

\section{$P A$ measurement}

We assessed PA using a questionnaire based on the Short Version of the International Physical Activity Questionnaire (6), which consists of three dimensional activity items: activity intensity level (light, moderate, and vigorous intensity), activity frequency per week, and activity time per day. We investigated PA at 2-time points: January 2020 (before the COVID-19 epidemic) and April 2020 (during the COVID-19 epidemic). The participants were required to provide 2 kinds of answers as a result of this investigation: recalled answer in January Published online June 23, 2020, http://10.1007/s12603-020-1424-2 
THE JOURNAL OF NUTRITION, HEALTH \& AGING

Table 1

The demographic variables and total PA time

\begin{tabular}{|c|c|c|c|c|}
\hline & $\begin{array}{c}\text { Overall } \\
n=1,600\end{array}$ & $\begin{array}{c}\text { Robust } \\
n=574\end{array}$ & $\begin{array}{c}\begin{array}{c}\text { Pre-frail } \\
\mathrm{n}=638\end{array}\end{array}$ & $\begin{array}{c}\text { Frail } \\
n=388\end{array}$ \\
\hline Age, mean (SD) & $74.0(5.6)$ & $73.6(5.4)$ & $73.9(5.7)$ & $74.7(5.9)$ \\
\hline Women, n (\%) & $800(50.0)$ & $320(55.7)$ & $284(44.5)$ & $196(50.5)$ \\
\hline Height, mean (SD) & $160.1(8.5)$ & $159.7(8.5)$ & $160.8(8.2)$ & $159.6(8.8)$ \\
\hline Weight, mean (SD) & $57.9(10.5)$ & $57.1(10.0)$ & $58.5(10.4)$ & $58.0(11.4)$ \\
\hline BMI, mean (SD) & $22.5(3.0)$ & $22.3(2.6)$ & $22.5(3.0)$ & $22.6(3.5)$ \\
\hline Lower health literacy, n \%) & $804(50.3)$ & $216(37.6)$ & $333(52.2)$ & $255(65.7)$ \\
\hline Polypharmacy, n (\%) & $315(19.7)$ & $80(13.9)$ & $117(18.3)$ & $118(30.4)$ \\
\hline \multicolumn{5}{|l|}{ Morbidity } \\
\hline Hypertension, n (\%) & $566(35.4)$ & 191(33.3) & $219(34.3)$ & $156(40.2)$ \\
\hline Hyperlipidemia, n (\%) & $212(13.3)$ & $69(12.0)$ & $85(13.3)$ & $58(14.9)$ \\
\hline Cancer, $\mathrm{n}(\%)$ & $92(5.8)$ & $26(4.5)$ & $40(6.3)$ & $26(6.7)$ \\
\hline \multicolumn{5}{|l|}{ Total PA time (minutes) } \\
\hline January-2020, median (IQR) & $245(90-480)$ & $330(148-600)$ & $270(120-480)$ & $123(0-360)$ \\
\hline April-2020, median (IQR) & $180(0-420)$ & $210(60-450)$ & $180(4-420)$ & $85(0-240)$ \\
\hline
\end{tabular}

SD: standard deviation, IQR: interquartile range, BMI: body mass index, PA: physical activity

situation and non-recalled answer in the current situation (April), respectively. We multiplied by activity frequency per week and activity time per day (minutes) at each activity level, and determined the total PA time (minutes) as added values for each level.

\section{Definition of the frailty category}

We assessed the frailty status with the Kihon checklist (7), which consists of 25 items (with yes/no responses) that are divided into seven domains: lifestyle (5 items), motor abilities (5 items), nutrition (2 items), oral functions (3 items), seclusion ( 2 items), forgetfulness ( 3 items), and emotions (5 items). We defined scores of 8 or more as frailty, 4 to 7 as pre-frailty, and 0 to 3 as robustness, according to a previous study (8).

\section{Demographic data}

The following 9 items were obtained: age, gender, height, weight, body mass index (BMI), living alone, number of medications, morbidity, and health literacy. We defined the number of medications; 5 or more as polypharmacy (9). We assessed the level of health literacy using the Critical Health Literacy (CCHL) scale (10), which includes 5 items (each item is rated on a 5-point scale: 1 being "strongly disagree" to 5 being "strongly agree"). We added each item and divided it by 5 and defined a score of less than 4 points as lower health literacy according to a previous study (10).

\section{Statistical analysis}

We examined the effect of the COVID-19 epidemic in the main and subgroup analyses for total PA time using the Wilcoxon rank-sum test. For subgroup analysis according to the three frailty categories, we divided the patients into three groups: robustness, pre-frailty, and frailty. The data were analyzed using SPSS (Statistical Package for the Social Sciences, Windows version 26.0; SPSS, Inc., Chicago, IL, USA). A P value less than 0.05 was considered to indicate statistical significance for all analyses.

\section{Results}

The demographic variables are listed in Table 1. The mean age, proportion of women, and mean BMI were $74.0 \pm 5.6$ years,

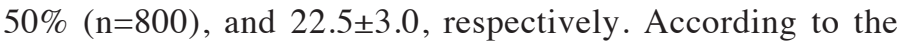
Kihon checklist, 388 participants $(24.3 \%)$ had a score of 8 or more (frail), 638 (39.9\%) had a score of 4 to 7 (pre-frail), and 574 participants (35.9\%) had a score of 0 to 3 (robust).

Table 1 and Figure 1 show the median total PA time in January 2020 and April 2020. Overall, we found a significant decrease in total PA time in April 2020 (median [interquartile range (IQR)], 180 [0 to 420]) when compared to January 2020 (median [IQR], 245 [90 to 480]) $(\mathrm{P}<0.001)$. We also performed a subgroup analysis according to the frailty category and found that the total PA time significantly decreased in April 2020 when compared to January 2020 for all frailty categories $(\mathrm{P}<0.001)$. 


\section{EFFECT OF COVID-19 ON PHYSICAL ACTIVITY}

\section{Figure 1}

Overall median total PA time in January 2020 and April 2020 (A), robust (B), pre-frail (C), and frail (D) participants. Overall, total PA time significantly decreased from January 2020 to April $2020(\mathrm{P}<0.001)(\mathrm{A})$. In a subgroup analysis according to the frailty category, total PA time significantly decreased in April 2020 when compared to January 2020 for all frailty categories $(\mathrm{P}<0.001)(\mathrm{B}, \mathrm{C}$, and $\mathrm{D})$

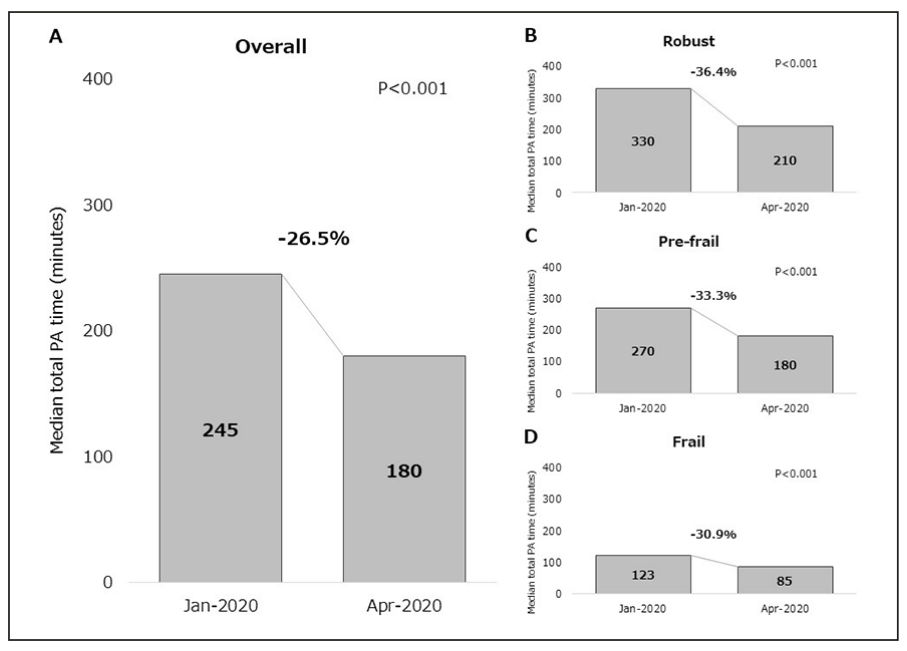

Discussion

Total PA time per week for older adults decreased by 65 minutes $(-26.5 \%)$ from January to April 2020. The total PA time in January and April 2020 was 330 and 210 minutes (-36.4) in robust older adults, 270 and 180 minutes (-33.3\%) in pre-frail older adults, and 123 and 85 minutes $(-30.9 \%)$ in frail older adults, respectively. The total PA time during the COVID19 epidemic in robust older adults was similar to baseline in pre-frail ones, and that in pre-frail ones was similar to baseline in frail ones. Therefore, the total PA time in older adults was reduced by one rank each by the COVID-19 epidemic.

It is a serious problem that the total PA time per week decreased by 65 minutes in older adults. A previous study indicated that daily step counts and light or moderate PA for Japanese older adults are lowest in the winter season (December to February), and highest in spring (March to May) or autumn seasons (September to November) due to the weather (11). In 2020 , the average mean temperature was $7.1{ }^{\circ} \mathrm{C}$ for January and $12.8{ }^{\circ} \mathrm{C}$ for April in Tokyo, Japan, respectively (12). Thus, normally, the total PA time must be higher in April than in January, and the change was much smaller than expected.

Contrary to our hypothesis, there were no differences in PA decline among the three frailty categories. We hypothesized that robust and pre-frail older adults may maintain their total PA time, but not frail older adults. However, the total PA time consistently decreased due to the COVID-19 epidemic across all frailty category groups. These results may suggest a downward spiral of lower physical function and disability in the near future. Thus, we must promote indoor exercise and walking habits around houses, and focus on the maintenance of healthy performance in older populations.

There are both strengths and weaknesses to this online survey. The strength of this survey was the elucidation of up to date status of PA in the older population. We believe that these findings can be useful to maintain healthy conditions and prevent disabilities in older adults worldwide. However, there was a sampling bias and lack of external validity in this survey because the study comprised participants who could use Internet connected devices and were registered with a survey company.

In conclusion, due to the COVID-19 epidemic, the total PA time in April 2020 was significantly lower than that in January 2020 in older adults. This finding may lead to a higher incidence of disability in the near future. Thus, we should assist older adults and promote indoor exercise and walking habits around the house.

Aknowledgment: This work was supported by the Research Fund for Longevity Science from the Grants-in-Aid for Comprehensive Research on Aging and Health from the Ministry of Health, Labor, and Welfare, Japan.

Disclosures: The authors have no potential conflicts of interest to disclose.

Conflict of interest: None

Ethical Standards: The ethics committee approved the study.

\section{References}

1. Fried LP, Tangen CM, Walston J, et al. Frailty in older adults: evidence for a phenotype.J Gerontol A Biol Sci Med Sci 2001; 56: M146-56.

2. Ensrud KE, Ewing SK, Taylor BC, et al. Comparison of 2 frailty indexes for prediction of falls, disability, fractures, and death in older women. Arch Intern Med 2008: 168: 382-9.

3. García-Hermoso A, Ramirez-Vélez R, Sáez de Asteasu ML, et al. Safety and Effectiveness of Long-Term Exercise Interventions in Older Adults: A Systematic Review and Meta-analysis of Randomized Controlled Trials. Sports Med 2020 [Epub ahead of print]

4. Saint-Maurice PF, Troiano RP, Bassett DR Jr, et al. Association of Daily Step Count and Step Intensity With Mortality Among US Adults. JAMA 2020; 323: 1151-1160.

5. Tomata Y, Suzuki Y, Kawado M, et al. Long-term impact of the 2011 Great East Japan Earthquake and tsunami on functional disability among older people: A 3-year longitudinal comparison of disability prevalence among Japanese municipalities. Soc Sci Med 2015; 147: 296-9.

6. Craig CL, Marshall AL, Sjöström M, et al. International physical activity questionnaire: 12-country reliability and validity. Med Sci Sports Exerc 2003; 35: 1381-95

7. Arai H, Satake S. English translation of the Kihon Checklist. Geriatr Gerontol Int 2015; 15: 518-9.

8. Satake S, Senda K, Hong YJ, et al. Validity of the Kihon Checklist for assessing frailty status. Geriatr Gerontol Int 2016; 16: 709-15.

9. Kojima T, Akishita M, Nakamura T, et al. Polypharmacy as a risk for fall occurrence in geriatric outpatients. Geriatr Gerontol Int 2012; 12: 425-30.

10. Ishikawa H, Nomura $\mathrm{K}$, Sato $\mathrm{M}$, et al. Developing a measure of communicative and critical health literacy: a pilot study of Japanese office workers. Health Promot Int 2008; 23: 269-74.

11. Yasunaga A, Togo F, Watanabe E, et al. Sex, age, season, and habitual physical activity of older Japanese: the Nakanojo study. J Aging Phys Act 2008; 16: 3-13.

12. Japan Meteorological Agency. http://www.data.jma.go.jp/obd/stats/etrn/index.php. Accessed May 2, 2020. 